\title{
INTERRELACION ENTRE LAS ESPECIES DE MICROALGAS HALLADAS EN EL ESTANY DE CULLERA (VALENCIA)
}

\author{
C. Rojo, M.R. Miracle, M. Serra \\ Dpto. de Ecología. Facultad de Biología. Universidad de Valencia.
}

Palabras clave: Coastal lagoon, phytoplankton, phytoplankton associations, Cullera lagoon (Spain).

\author{
ABSTRACT \\ INTERRELATION BETWEEN THE MICROALGAL SPECIES IN THE COASTAL LAGOON OF \\ CULLERA (VALENCIA, SPAIN)
}

A statistical study in order to characterize the groups of association of phytoplankton species of a meromictic coastal lagoon has been carned out.

In the analyses were use a total of 120 species selected because their ocurrence from the 175 ones identified in the samples taken at different depths and at different hours ofthe day, during an annual cycle, in three sampling stations located at different distances from the sea. The results show that hours of the day and distance from the sea did not have much influence in the clustering of species. The main groups of association correspond principally to succesional stages in the annual cycle and slightly to vertical distribution due to the remarkable stratification of the lagoon.

The best defined phytoplankton associations were following: (1) A group (G-1) composed mainly by small centric diatoms, $C y$ clotella atomus and several pennate diatoms of the genus Nitzschia, which can be considered as one of the first succesional stages. (2) A group (G-1) formed by cryptomonads as Cryptomonas ovata and Rhodomonas minuta and several chlorophyta which succeeds and replaces G-1 in early summer. (3) Several groups are more or less characteristics of summer being the most important G-IV, which is composed by cianobacteria, chysophycea and one or two species of euglenophyta dinoflagellatesand crytomonads; the latter represents the more advanced succesional stage in the lagoon. And (4) another group made up whith small centric diatoms, characteristic of fluctuating conditions, which takes place in winter when there is a strong marine influence. The main species of this group is the brackish water Stephanodiscus subsalsus which is associated with several species having a tendency to occur in the chemocline, either because they require high salinity such as Skeletonema costatum or because they can live in anoxic conditions such as Astasia inflata and Lyngbva limnetica.

\section{INTRODUCCION}

El Estany Gran de Cullera, situado a unos $42 \mathrm{Km}$. al sur de la ciudad de Valencia, es una laguna litoral alargada, perpendicular a la costa, de unos $3,5 \mathrm{Km}$. de longitud por 0,4 de ancho. Se comporta como un estuario y presenta una intrusión de agua marina por el fondo de condiciones anóxicas y reductoras.

Las particularidades de esta laguna debidas a la estratificación de sus aguas, especialmente la distribución en el perfil vertical de los pigmentos fotosintéti$\cos$ y actividad fototrófica de algas y bacterias en el punto más profundo, ha sido descrita por Miracle y Vicente $(1983,1985)$. Una relación de las especies fi-

Limnética 2: 3540 (1986)

O Asociación Española de Limnología. Madrid. Spain toplanctónicas correspondientes a los mismos muestreos que sirvieron de base al citado trabajo, se puede encontrar en Rojo y Miracle (1985), estudio que especifica además la importancia en el plancton de cada una de dichas especies y la variación estacional de las principales. Estos estudios eran parte de un trabajo mas amplio que comprendía además otros puntos de muestreo mas o menos alejados del mar y ciclos diarios dentro del mismo ciclo anual.

El presente trabajo consiste en la elaboración estadística de todos los datos del fitoplancton obtenidos en el Estany de Cullera correspondientes a tres estaciones de muestreo, diferentes profundidades y distintas horas del día y épocas del año. Su objetivo princi- 


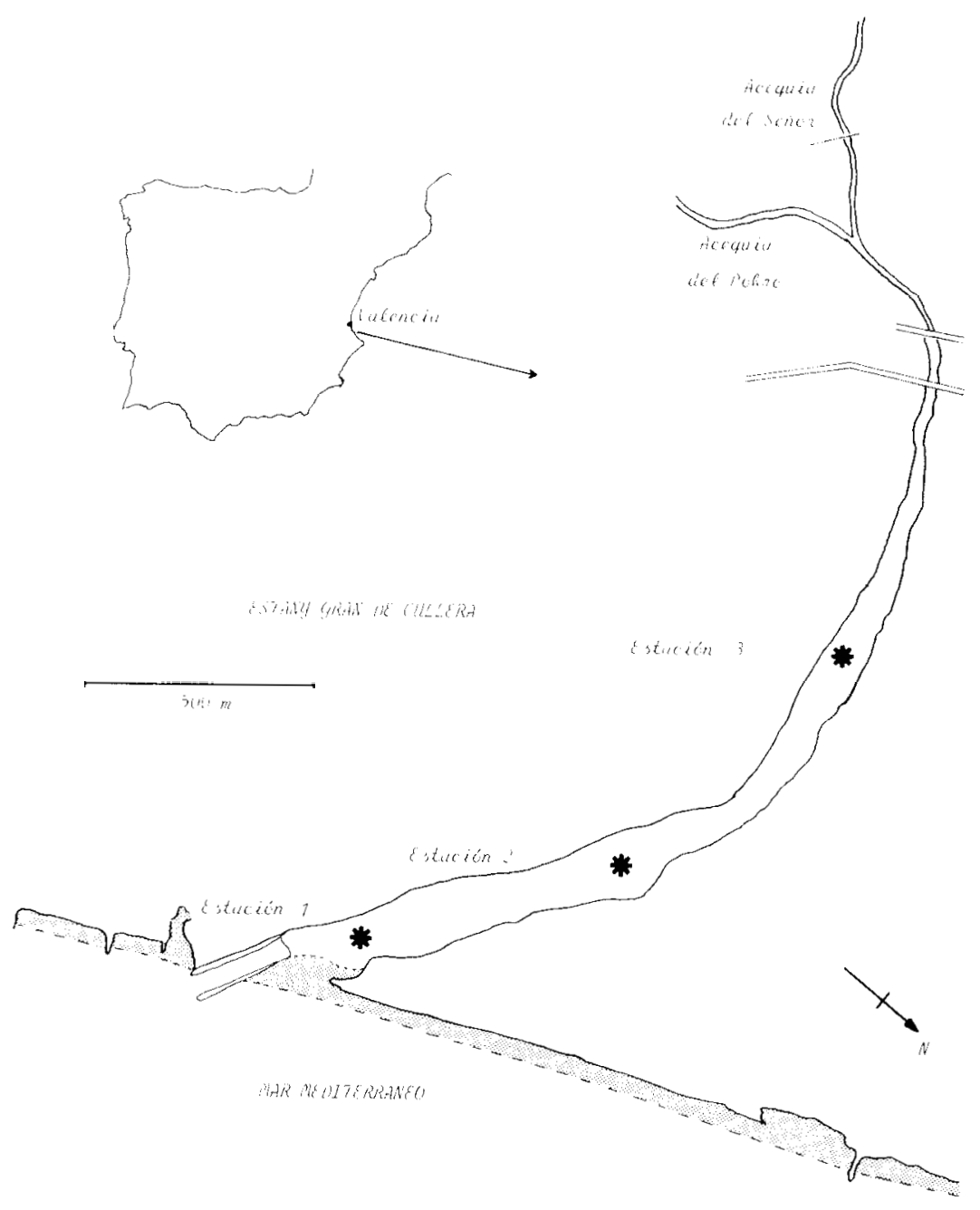

Figura 1.-Esquema del Estany de Cullera, donde se indican los tres puntos de muestreo.

Outline of Cullera lagoon indicating sampling stations.

pal es el de buscar las posibles asociaciones de algas, que puedan caracterizar los distintos subambientes espacio-temporales de la laguna.

\section{MATERIAL Y METODOS}

El periodo de estudio comprende desde el mes de agosto de 1980 hasta octubre de 1981, tomándose muestras en nueve ocasiones correspondientes a diferentes épocas del año.

Los muestreos se hicieron en tres localidades (figura 1): (1) cerca de la desembocadura en el mar, que presentaba $5 \mathrm{~m}$. de profundidad, (2) en la fosa central que presentaba la profundidad máxima de 7 m., y (3) cerca de la entrada de la acequia de agua dulce, con una profundidad de $3 \mathrm{~m}$. En el punto central se repitieron a diferentes horas del día. Se obtuvo un total de 141 muestras recogidas a diferentes profundidades según la localización de la termoclina en dichos puntos de las que $21,103,17$ corresponden respectivamente a las localidades 1,2 y 3 .

Las muestras fueron recogidas con una bomba peristáltica conectada a un dispositivo en forma de doble cono situado a la profundidad deseada, y fijadas con lugol. Para efectuar el recuento de las especies del fitoplancton se utilizaron cubetas de $50 \mathrm{ml}$. y un microscopio invertido Olympus (Utlermohl, 1958).

Para estudiar los grupos de asociación entre especies se efectuaron varios análisis de agrupamiento o 
«clusters» mediante el método UPGMA (Sneath y Sokal, 1973), partiendo de la matriz de correlación entre especies, caracterizadas por el número de individuos por ml. que presentaban en cada muestra, previa transformación logarítmica. Anteriormente se seleccionaron aquellas especies que se habían encontrado por lo menos en dos de las muestras. Concretamente, se efectuó un análisis de agrupamiento incluyendo el total de muestras (141 casos, 120 variables), otro utilizando sólo las muestras recogidas por encima de la quimioclina, es decir, en el mixolimnion (75 casos y 119 variables) y un tercero utilizando las recogidas en la quimioclina y por debajo de la misma (66 casos. 49 variables).

\section{RESULTADOS}

Se identificaron un total de 175 especies en las muestras obtenidas. la mayor parte de las cuales figuran en la relación ya publicada en el citado trabajo (Rojo y Miracle, 1985) con los datos de la estación de mucstreo 2. En el análisis de agrupación se utilizaron sólo 120 especies de dicho total al no considerar aquellas que se habían encontrado en una Única muestra.

Los resultados del análisis de muestras eran prácticamente iguales a los obtenidos con sólo las muestras del mixolimnion, por ello nos centramos tan solo en el análisis con el total de muestras para discutir las asociaciones algales. Sin embargo, el análisis con las muestras de la quimioclina y monimolimnion aportaba una mayor información sobre algunos grupos de especies menos destacados en los anteriores resultados, especialmente los constituidos por algas adaptadas a salinidades más elevadas y que aguantan condiciones de escasez de oxígeno.

Los resultados del análisis general con la totalidad de las muestras se presentan en la hemimatriz sombreada de la figura 2 , en donde se diferenciaron siete grupos que corresponden a las distintas etapas sucesionales y que tienen una clara preferencia por situarse en las distintas zonas del perfil vertical, determinadas por la importante estratificación de las aguas (figura 4). Durante el periodo de estudio dicha estratificación estuvo definida en verano y otoño por un gradiente dc salinidad muy marcado entre los 3 y $4 \mathrm{~m}$. de profundidad que coincide con la interfase oxigeno-sulfhídrico o quimioclina. Esta estratificación se deshizo en parte a finales de otoño-invierno, de manera que la quimioclina quedó situada a mayor profundidad entre 4 y $5 \mathrm{~m}$. y el gradiente de salinidad fue mucho menos marcado situándose entre 1 y $2 \mathrm{~m}$. de profundidad.

Un primer grupo, G-I, está formado por diatomeas céntricas que caracterizan las primeras etapas sucesionales y aparecen sobre todo en primavera, presen- tando algunas de ellas crecimientos masivos (concretamente, Cyclotella atomus cuyo número constituyó más del $80 \%$ del fitoplancton a principios de mayo). Estas diatomeas van acompañadas de clorofíceas pequeñas y algunas diatomeas pennadas, principalmente del género Nitzschia (figura 2). Esta asociación aparece preferentemente en primavera ṕero perdura hasta mediados de verano; se sitúa en aguas superficiales y se relaciona con los otros grupos de asociación, pues especies pertenecientes a estos ultimos presentan también correlaciones altas con algunas del grupo $\mathrm{G}-\mathrm{I}$.

El grupo G-II está constituido principalmente por criptofíceas, como Rhodomonas minuta y Cryptomonas ovata, y algunas clorofíceas. Este grupo es característico de verano y de aparición posterior al G-I ya mencionado, al que sucede en el tiempo. La aparición de las criptoficeas ocurre por regla general durante el periodo de estratificación, permitiendo sus características de mayor motilidad y tamaño su desarrollo en etapas algo avanzadas de la sucesión anual. El G-IV está constituido principalmente por cianoficeas y crisofíceas acompañadas también por algunas criptoficeas y en menor medida, por alguna dinoflagelada y euglenofícea. Este grupo sucede al anterior, caracterizando una etapa más avanzada. Esta asociación aparece en el Estany de Cullera durante un periodo de tiempo más corto. Principalmente a principios de otoño, estación del año durante la cual persiste la estratificación del agua.

Otro grupo bien definido es el G-V, que está formado por diatomeas céntricas de pequeño tamaño, crecimiento rápido y que aparecen en situaciones de grandes cambios ambientales. Su aparición coincide con el primer periodo de mezcla vertical de la laguna en invierno, el cual lleva aparejado un aumento de salinidad en gran parte de la columna que favorece especies características del litoral marino, como Skeletonema costatum que tiende a presentarse en profundidad donde la influencia marina es mayor. El representante principal del grupo es Stephanodiscus subsalsus, que presentó una gran proliferación en el periodo invernal de mezcla, con una fuerte dominancia respecto a las demás especies del fitoplancton.

El resio de las asociaciones no tienen interpretaciones tan claras. Se pdne de manifiesto un grupo de verano y superficial formado por Pediastrum borvanum y Oscillatoria tentis (Grupo VII). Por otra parte, en la matriz sombreada se observan dos grupos de especies más bentónicas, el grupo G-III, y el grupo G-IV, el primero de ellos está formado por diatomeas bentónicas con máximos a finales de verano, que tienden a situarse a mayor profundidad. El grupo $G-V I$ es poco claro, si bien se observa una tendencia de las especies más representativas a ocupar niveles algo profundos, siendo su presencia primaveral. 


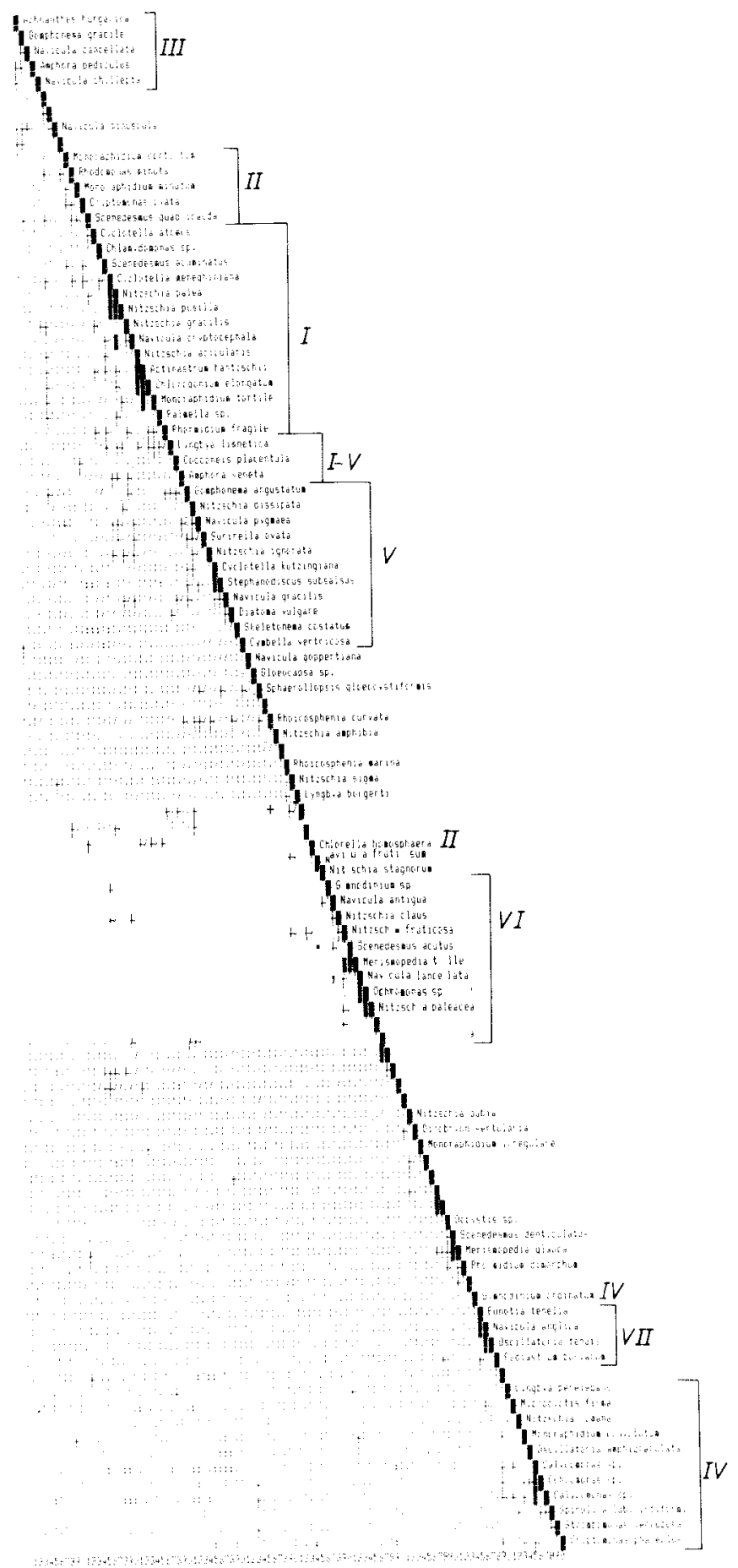

Figura 2.-Hemimatriz sombreada de los coeficientes de correlación (reordenada por el análisis de agrupamiento) entre las especies de ocurrencia mayor que 1 y calculada con el total de las muestras. Sólo las especies que presentaban coeficientes de correlación superiores a 0.4 han sido representadas.

Shaded correlation matrix (rearranged by cluster analysis) between phytoplanktonic species (ocurence 1) computed with the 1otal number of samples indicating ttic cluster of species. Only species showing coefficients greater than 0.4 have been included. 


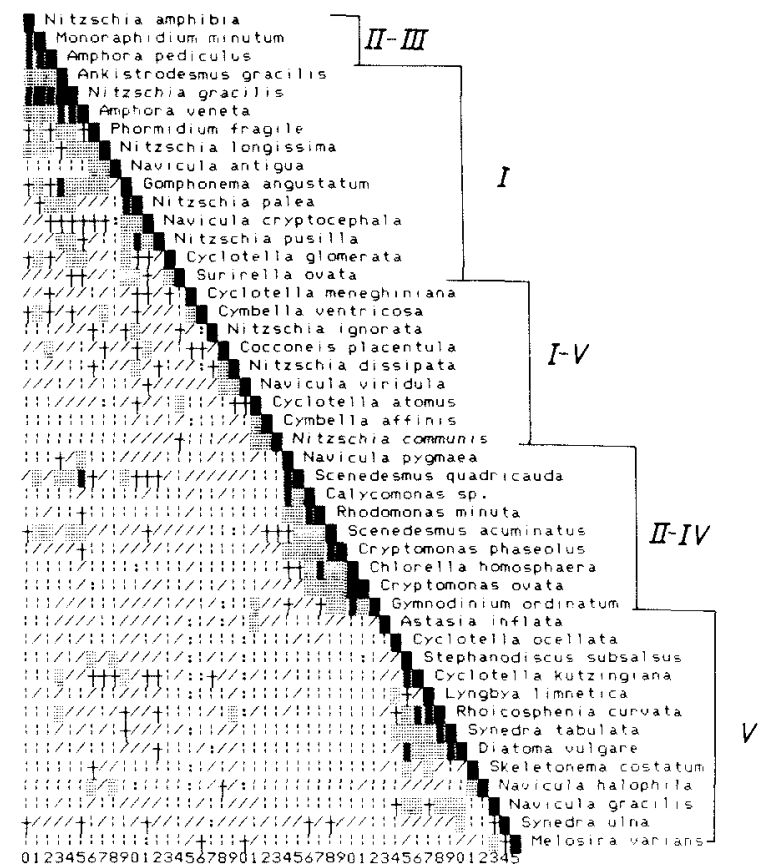

Figura 3.-Hemimatriz sombreada de los coeficientes de correlación (reordenada por el análisis de agrupaniierito) entre las especies de ocurrencia mayor que 1 y calculada con las muestras de la quimioclina y por debajo de ella. Sólo las especies que presentaban coeficientes superiores a 0.4 han sido representadas.

Shaded correlation matrix (rearranged by cluster analysis) between phytoplanktonic species (ocurence 1) coinputing only samples from and below the chemocline indicating the cluster of species. Only species showing coefficients grenter than 0.4 have been included.

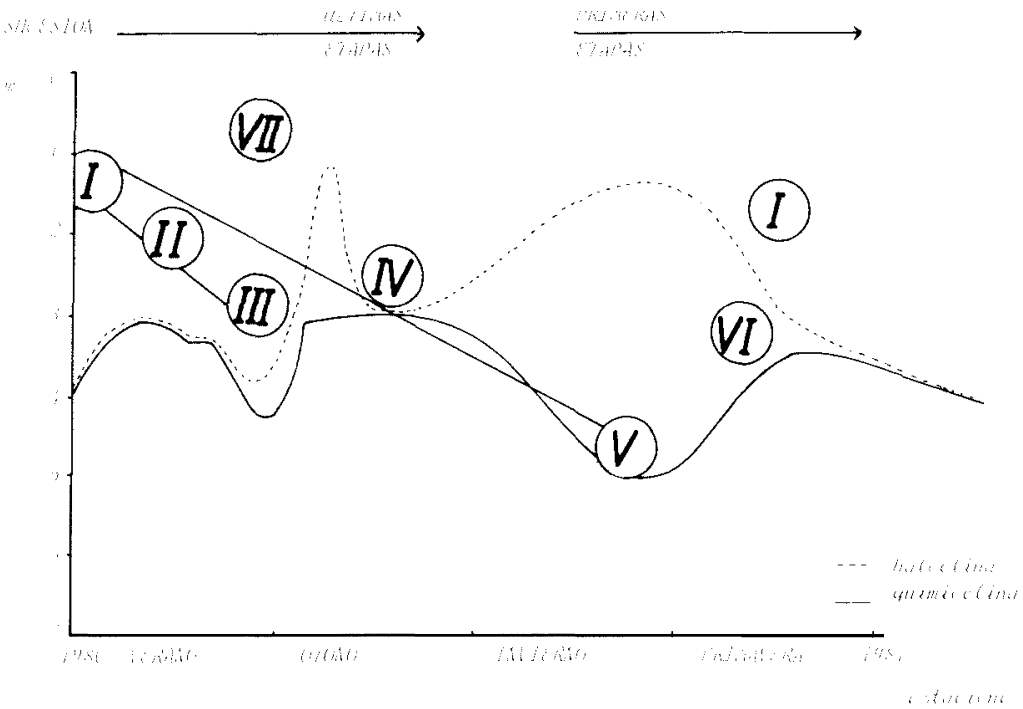

Figura 4.-Distribución de los grupos de asociación de las especies del fitoplancton en relación con la estratificación de las aguas y ciclo anual. Los grupos se han unido con una línea si presentaban conexiones entre ellos. Se indica además su relación con las distintas etapas de la sucesión y estaciones del año.

Distribution of the clusters or groups of species in relation with uater stratification and annual cycle. The groups havc been connected by lincs wlien they related. The correspondence ofthisgroups with successional stages is also indicated. 
Los resultados del análisis con las muestras de la quimioclina y monimolimnion se recogen el la figura 3 , donde se indican los grupos de especies anteriormente definidos, ya que en general se da una coincidencia de las especies asociadas, aunque se observan algunas diferencias: las especies de los grupos VI y VII no se encuentran en estas muestras salvo alguna excepción. Faltan la mayoría de clorofíceas que integraban los grupos G-I y G-II. No aparecen las especies de crisofíceas y cianofíceas de los grupos G-II y G-IV, fundiéndose ambos grupos en uno solo.

Sin emburgo, en la figura 3 el grupo G-V ha quedado más compacto y mejor definido que en el análisis de todas las muestras (figura 2). Por ello el grupo G-V podría considerarse que representa aquellas especies algales que pueden vivir en la quimioclina. En este grupo se reúnen especies como Stephanodiscus subsalsus, que presenta grandes crecimientos en la superficie, pero también se acumula en la quimioclina con especies que pueden encontrarse en las capas anóxicas profundas, como Astasia inflata y Lyngbya limnetica junto con bacterias fotosintéticas del azufre (Miracle y Vicente, 1985).

\section{DISCUSION}

El fitoplancton del Estany de Cullera, que recibe aportes de agua dulce y salada, presenta grandes variaciones en el tiempo (Rojo y Miracle, 1985) debidas a las importantes fluctuaciones de los parámetros ambientales, que determinan unas asociaciones entre especies fitoplanctónicas correspondientes sobre todo con las distintas épocas del año. Por el contrario, no ha aparecido ningún grupo de asociación de especies característicc de alguna de las localidades de mues$\mathrm{t} r$ e a lo largo de la laguna. debido en parte a la escasa longitud de la misma y a que la influencia de agua marina llega hasta la localidad muestreada más distante del mar.

Dada la importante estratificación de las aguas, se establecen algunos grupos que tienden a una distribución diferenciada en el perfil vertical. No obstante, la variación debida a la profundidad es mucho menor que la debida a las estaciones del año. Las aguas de la quimioclina y monimolimniom están dominadas absolutamente por bacterias fotosínteticas del azufre (Miracle y Vicente 1985), entre las que aparecen en escaso número algas qlie están tambien presentes en las aguas más superficiales algunas son precisamente las que presentan máximos en una época determina$\mathrm{da}$, en especial en las que hay una mayor turbulencia y mezcla de aguas, por lo que su presencia en las aguas anóxicas es accidental. Sólo determinadas especies de cianobacterias y euglenales podrían considerarse como pobladoras de las aguas anóxicas. En la quimioclina podrían concentrarse tambien especies características de aguas más saladas o marinas y presentar su máximo cerca de dicha interfase.

El análisis estadístico ha configurado sobre todo asociaciones entre especies que corresponden a una sucesión de comunidades en el tiempo (Fig. IV). Las épocas invernales son de influencia marina, lo que favorece la entrada de especies de agua más salada o de distribución marina. como Skeletonetna costattim. y provoca un crecimiento explosivo de especies de agua salobre, como Stephanodiscus subsalsus. Estas especies se agrupan en un grupo de asociación, muy claro que reúne especies de aguas saladas y otras que toleran condiciones anóxicas (Grupo V).

Desde finales de primavera domina el flujo de agua dulce, estableciéndose en verano una estratificación muy marcada entre una capa de agua dulce y una de agua salada. Durante los meses cálidos del año tiene lugar una sucesión propiz de un lago eutrófico con una proliferación en primavera de Cyclotella atomus acompañada de otras diatomeas, algunas pennadas que se encuentran en el plancton superficial debido a la turbulencia y circulación vertical de las aguas en esta época (Grupo I). Este grupo es sustituido en verano y otoño por otros en que abundan más las clorofíceas, al principio, y, más adelante las criptofíceas, cianofíceas y dinoflageladas (G-II, G-IV, G-VII).

Variaciones temporales parecidas, determinadas por regímenes de influencia marina invernal y periodos de estabilidad en verano-otoño en los que progresa una sucesión planctónica, han sido observados también en otras lagunas costeras del Mediterráneo español (Comín 1984), con flujo de agua dulce más o menos controlado por el cultivo del arroz en los campos circundantes.

\section{BIBLIOGRAFIA}

Comín, F.A. 1984. Características físicas y químicas y fitoplancton de las lagunas costeras, Buda, Encañizada y Tancada (Delta del Ebro). Oecología aquatica Vol. 7, 79-162.

Miracle, M.R. y Vicente, E. 1983. Vertical distribution and rotifer concentrations in the chemocline of meromictic lakes. Hydrobiologia 104, 259-267.

Miracle, M.R. y Vicente, E. 1985. Phytoplankton and photosynthetic sulphur bacteria production in the meromictic coastal lagoon of Cullera (Valencia. Spain). Verh. Internat. Verein. Limnol. 22: 2214-2220.

Rojo, C. y Miracle, M.R. 1984. Fluctuación estacional de las comunidades fitoplanctónicas del Estany de Cullera (Valencia). Anales de Biología (Univ. de Murcia) (2): 161-168.

Sneath, P.H.A. y Sokal, R.R. 1973. Numerical taxonomy. Freeman. San Francisco Utlermohl, H. 1958. Zur Vervollkommung der quantitative Phytoplankton-Methodik. Mitt. Int. Verein. Lirnnol. 9: 1-38. 